\title{
Tandem cylinder flow and noise predictions using a hybrid RANS/LES approach
}

\author{
M. WEINMANN ${ }^{\mathrm{a}}$, R. D. SANDBERG ${ }^{\mathrm{a}, *}$, C. DOOLAN ${ }^{\mathrm{b}}$ \\ ${ }^{a}$ Aerodynamics and Flight Mechanics Research Group, Faculty of Engineering and \\ the Environment, University of Southampton, Southampton SO17 1BJ, U.K. \\ ${ }^{\mathrm{b}}$ School of Mechanical Engineering, The University of Adelaide, South Australia, \\ Australia 5005
}

\begin{abstract}
The performance of a novel hybrid RANS/LES methodology for accurate flow and noise predictions of the NASA Tandem Cylinder Experiment is investigated. The proposed approach, the modified Flow Simulation Methodology (FSM), is based on scaling the turbulence viscosity and the turbulence kinetic energy dissipation rate with a damping function. This damping function consists of three individual components, a function based on the Kolmogorov length-scale ensuring correct behavior in the direct numerical simulation (DNS) limit, a function ensuring that FSM provides the correct damping in large-eddy simulation (LES) mode, and a shielding function that forces the switch from Reynolds-averaged Navier-Stokes (RANS) to LES to occur outside the boundary layer. The FSM is proposed for the $k \omega$ SST two-equation model (FSM-SST) and for an Explicit-Algebraic-Stress-Model (FSM-EASM), which is better suited to resolve anisotropy and non-equilibrium of the unresolved scales and the strain and rotation-rate dependent coefficients introduce a dynamic response of the model to the resolved flow field. Simulations are performed on a relatively coarse grid and the FSM data are compared with results obtained from the Scale-Adaptive-Simulation (SAS) and IDDES approaches. Acoustic predictions are obtained using an acoustic analogy approach based on Curle's theory. The FSM-SST approach was found to predict the hydrodynamic field in very good agreement with reference data, whereas the FSM-EASM did not improve the predictions. The acoustic spectra predicted show good agreement with experimental results at various microphone positions, with some deficiencies in capturing the broadband noise levels at high Strouhal numbers.
\end{abstract}

Key words: hybrid RANS/LES methods, aeroacoustics, tandem cylinder, acoustic analogy, explicit algebraic stress models

* Corresponding author
Email address: sandberg@soton.ac.uk (, R. D. SANDBERG). 


\section{INTRODUCTION}

The engineering approach to perform turbulent flow simulations at high Reynolds number is typically based on the Reynolds-Averaged Navier-Stokes equations (RANS). At this level of turbulence closure, simulations can be performed for complex geometries with short turnaround times and at reasonable computational cost. Unfortunately, the RANS approach is limited in accuracy for more complex flows which depart from the inherent modeling and calibration assumptions, such as the bluff-body flow separation in question here.

In cases where a more accurate description of the flow is required, such as to study the detailed physics associated with turbulent flows or the aerodynamic noise produced by the flow, the RANS approach is not suited and one has to resort to more accurate approaches like Large-Eddy-Simulation (LES). The superior performance of LES stems from the capability to explicitly resolve the larger scales of turbulence which significantly contribute to the transport of mass, momentum and energy in the flow. However, the requirement that the large scales and energy producing events need to be represented explicitly in the simulation makes the computational cost of LES prohibitively high for high Reynolds number wall bounded flows. Therefore, LES cannot yet be considered a substitute for the RANS approach in engineering or industrial applications.

To overcome the limitation of LES in wall bounded flow, so-called hybrid RANS/LES modelling approaches have been proposed, such as the well-known DES[1], in which a RANS-based approach is used in the vicinity of the wall and coupled to an LES method away from it. A number of different hybrid RANS/LES methods exist by now, see, for example, the reviews by Sagaut et al.[2] or Fröhlich \& von Terzi[3].

Suggesting a unified RANS/LES approach at roughly the same time as the original DES formulation, Speziale[4] argued that a hybrid RANS/LES method should adhere to at least the following three properties: 1) The subgrid-scale model should be able to resolve anisotropy of the flow and allow for a direct integration to the wall without using empirical damping functions, 2) In the limit of a very coarse mesh or infinite Reynolds number the hybrid method needs to revert to a state-of-the-art RANS turbulence model, 3) Absence of any test filters or double-filtered fields. The first two points seem particularly important, since most subgrid-scale models are derived based on the assumption that the unresolved subgrid scales are isotropic. This assumption is not justified, for example, close to the wall where subgrid scales are likely to exhibit anisotropy. In addition, subgrid-scale models are not able to model a significant amount of Reynolds-stress; if the resolution becomes too coarse to explicitly resolve roughly $80 \%$ of the turbulence kinetic energy, subgrid-scale 
models are not adequate and results will deteriorate.

To satisfy these properties, Speziale[4] introduced a hybrid RANS/LES approach in which the unresolved stress components can be computed by damping the Reynolds-stress tensor predicted from a state-of-the-art RANS turbulence model

$$
\tau_{i j}=F \cdot \tau_{i j}^{R A N S} .
$$

The damping function $F$ allows for a continuous blending between RANS and DNS, depending on the local and instantaneous grid resolution. In general, the blending function needs to be designed such that $F \rightarrow 0$ if the grid resolution is sufficient to resolve all scales of turbulence, resulting in DNS. In the coarse grid or infinite Reynolds number limit, $F$ needs to approach unity, resulting in a RANS closure. For values $0 \leq F \leq 1$, the model is able to operate in a nontraditional LES mode. Note, the idea of rescaling the Reynolds stress tensor could equivalently be expressed as rescaling the turbulent viscosity as $\nu_{T}=\alpha \nu_{T}^{R A N S}$, see Batten et al.[5]. Since this hybrid approach is distinctly different from other RANS/LES methods, it was referred to as Flow Simulation Methodology (FSM) by subsequent authors[6,7]. One of the issues with the FSM approach is that of consistency, which was not addressed by Speziale[4] or subsequent FSM-based publications and deals with how the Reynolds-stress tensor $\tau_{i j}^{R A N S}$ is computed in case of resolved turbulence, where $\tau_{i j} \ll \tau_{i j}^{R A N S}$. In case a considerable amount of turbulence is resolved in the simulation, the computed flow variables are filtered quantities and not equivalent to a Reynolds-averaged quantity. Strictly speaking, Reynolds-averaged quantities are required for the RANS equations in order to be consistent in the computation of the Reynolds-stress tensor. This raises the fundamental question of which quantities should be used to compute the Reynolds-stress tensor in case of resolved turbulence[8].

The original damping function[9] was based on the ratio of grid spacing to Kolmogorov length scale $\Delta / L_{\eta}$

$$
F=f_{\eta}=\left[1-\exp \left(\frac{-\beta \Delta}{L_{\eta}}\right)\right]^{n},
$$

with $L_{\eta}$ the Kolmogorov length scale, $\Delta$ a grid scale, and $\beta$ and $n$ free parameters. Fasel et al. [10] applied FSM in its original form to a backward facing step, subsonic plane wake and a supersonic axisymmetric wake. The optimum value of $\beta$ was found to be approximately $\beta=0.001$ for $n=1$. However, those choices were ad-hoc and the functional shape of the damping function and the values of the constants $\beta$ and $n$ never completely justified.

Another issue with FSM is that the damping function based on $\Delta / L_{\eta}$ is able 
to provide the correct behavior in the DNS limit, since the Kolmogorov length scale is the relevant length scale in DNS. However, $L_{\eta}$ is not the relevant length scale for LES and using this length scale does not guarantee the correct amount of damping in LES mode. Therefore, at least one additional element has to be introduced to the damping function such that FSM provides LES capabilities.

As the grid resolution becomes too coarse to explicitly resolve the large scale and energy producing structures in the flow field, the grid spacing $\Delta$ cannot be considered as an adequate measure of the turbulence length scale to be used in the constitutive model equations. For coarse grid LES or very largeeddy simulation (VLES) the length scale has to approach the RANS turbulence length scale $L_{T}$. Most hybrid RANS/LES approaches, like for example, DES do not fall back to the RANS length scale $L_{T}$ and therefore require an LES-like resolution in regions where the model operates in LES mode. This requirement does not agree well with the reality of industrial applications, where the mesh quality and resolution is sometimes sacrificed for short turnaround times. One approach to obtain a better suited characteristic length-scale for coarse grid LES is to compute the length scale from a transport equation for the subgrid dissipation rate $\epsilon_{s g s}$ such as, for example, Scale-Adaptive Simulation[11] or Partially-Integrated-Transport-Model[12,13]. Alternatively, the hybrid RANS/LES framework could be based on a more sophisticated underlying RANS model that is able to predict non-equilibrium and anisotropy effects of the unresolved large scales. This belief is grounded in that non-linear and differential subgrid-scale models have been shown to improve mean-velocity predictions and second-order statistics[14,15]. Marstropp et al. [16] proposed an explicit algebraic subgrid-stress model and found that for coarse resolution the mean and statistical quantities were improved over results from the dynamic Smagorinsky model, while showing less sensitivity to the resolution of the computational grid. Moreover, these features may not only become important in the LES region, they are also very desirable for an accurate representation of the flow in the RANS region close to the wall, as recently demonstrated by Ashton et al.[17] in the context of DDES based on elliptic relaxation. The RANS model chosen in the current work is an Explicit-Algebraic-Stress-Model (EASM), which is, unlike models based on a linear stress-strain relationship, able to resolve anisotropy and non-equilibrium of the unresolved scales. In addition, its strain and rotation-rate dependent coefficients introduce a dynamic response of the model to the resolved flow field. Therefore, it is appealing to investigate the suitability of EASM in the context of hybrid RANS/LES modeling.

In order to assess the performance of hybrid RANS/LES methods, challenging test cases for which reliable benchmark data is available are required. One such test case is the prediction of the flow and noise field of a tandem cylinder configuration. The noise generated by tandem cylinder flows, classed as a problem of wake interference[18], is important because it occurs in a wide variety of ap- 
plications that include aircraft landing gears, industrial heat exchangers and many architectural situations. Despite a relatively simple geometry, facilitating mesh generation, the flow field is highly complex. The separation between the cylinders sets the type of wake interaction. When the cylinders are close to one another, vortex shedding from the upstream cylinder has been found to be suppressed. As the cylinder spacing is increased, a variety of flow modes are encountered, with upstream shear layer reattachment occurring first on the downstream cylinder, followed by the re-establishment of vortex shedding behind the upstream cylinder. Once this occurs, the impingement of the wake on the downstream body creates high amplitude unsteady forces and intense radiated noise. This is usually classified as the co-shedding[19] or critical[20] regime. A review of the fluid mechanics of flow over pairs of cylinders in tandem, side-by-side and staggered arrangements is given by Sumner[21].

A recent experimental program has been performed at high Reynolds number $\left(1.66 \times 10^{5}\right)$ by NASA[22-24] (termed the NASA Tandem Cylinder Experiment in this paper) that provides a comprehensive data set for aeroacoustic simulation validation. The experimental reference data have been used extensively in various efforts for assessment and improvement of numerical flow and noise prediction tools[20,25-33]. In addition, the tandem cylinder configuration was used as a benchmark case in the AIAA workshop on Benchmark problems for Airframe Noise Computations (BANC I/II) [34,35] and in the 7th EU framework project Advanced Turbulence simulation for Aerodynamic Application Challenges (ATAAC) [36,37]. Thus, a wide range of simulation results are available for comparison of the data with different codes, turbulence models and spatial/temporal discretizations.

The current paper aims to investigate in detail:

- Assessment of a modified FSM approach for the flow and noise prediction of a tandem cylinder configuration. The performance of the novel methodology is compared with results from established approaches like the IDDES[38] and SAS[39].

- Importance of the baseline RANS model in the hybrid RANS/LES framework. This issue is investigated by comparing the performance of FSM approaches based on both the $k$ - $\omega$-SST model and the $k-\omega$-EASM model. In particular, the performance for coarse grids in the LES region is investigated. 


\section{METHODOLOGY}

\subsection{The Modified Hybrid RANS/LES Model Formulation}

The success of the FSM approach hinges on the quality of the damping function $F$. As mentioned in the introduction, the original damping function of Speziale[9] was based on the ratio of grid spacing to Kolmogorov length scale $\Delta / L_{\eta}$ and can only be expected to provide the correct behaviour in the DNS limit. Using the Kolmogorov length scale, there is no guarantee that this damping function will provide the correct magnitude of damping in LES mode. In the current paper, several modifications have been made to the original damping function in order to obtain better overall performance of the FSM. To ensure that the modified FSM approach still provides the correct behavior in the DNS limit, the original damping function has been retained.

The first modification ensures that FSM provides the correct damping in LES mode. LES requires a grid resolution such that the resolution cut-off is located in the inertial subrange of the energy spectrum. In LES the grid size can be used as the characteristic length scale of the unresolved turbulence. Therefore, an additional parameter $f_{\Delta}=C_{\alpha} \Delta / L_{T}$ is introduced to the damping function $F$, where $C_{\alpha}$ is a calibration constant. The filter length scale is defined in this work as the cube root of the cell volume, i.e. $\Delta=\mathrm{d} V^{1 / 3}$, which is suitable for cells of arbitrary polyhedral shapes.

If the filter width is smaller than the local characteristic length scale, $C_{\alpha} \Delta<$ $L_{T}$, the FSM approach will operate in LES mode, with $F<1$. This usually occurs away from the wall where the grid size is sufficiently fine to resolve turbulence fluctuations. As the wall is approached, the length scale $L_{T}$ goes to zero, since the turbulence kinetic energy vanishes at the wall, $k_{w}=0$. As a consequence, $C_{\alpha} \Delta>L_{T}$ close to the wall, which gives $F>1$. To avoid that the damping function exceeds unity, $F=\min \left[f_{\eta} f_{\Delta}, 1\right]$ is used.

This modified FSM formulation still suffers from the same grid sensitivity issues as the original DES formulation [11]. It is therefore essential to introduce another element, $f_{z}=1 /\left(1-F_{z}\right)$, to the damping function $F$ that ensures the switch from RANS to LES occurring outside the boundary layer. $F_{z}=F_{1}$, where $F_{1}$ is the blending function from the underlying $k$ - $\omega$ SST turbulence model described in the next section. The final formulation of the new damping function, including a boundary-layer correction, is therefore

$$
F=\min \left[f_{\eta} f_{\Delta} f_{z}, 1\right]=\min \left[\left(1-\exp \left(-\frac{\beta \Delta}{L_{\eta}}\right)\right)^{n} \frac{C_{\alpha} \Delta}{L_{T}}\left(\frac{1}{1-F_{z}}\right), 1\right]
$$


With a new formulation of the damping function available, it is important to decide what terms to actually damp in order to address the issue of consistency, raised in the introduction. The question of consistency can be understood using analysis of the turbulence viscosity and turbulence kinetic energy dissipation rate, which are given by $\nu_{T}=C_{\mu} V_{T} L_{T}$ and $\epsilon=C_{\epsilon} V_{T}^{3} / L_{\epsilon}$, where $L_{T}$ and $L_{\epsilon}$ are two characteristic turbulence length scales and $V_{T}$ is a characteristic velocity scale of the largest unresolved scales of motion. Using the Boussinesq approximation, the FSM approach can be written as

$$
\overline{u_{i}^{\prime} u_{j}^{\prime}}=F \cdot{\overline{u_{i}^{\prime} u_{j}^{\prime}}}^{R A N S}=(2 / 3) F k \delta_{i j}-2 F \nu_{T} \bar{S}_{i j}^{*}
$$

The turbulence kinetic energy $k=V_{T}^{2}$ in (4) is sensitive to the resolved flow field through the velocity gradient in the production term $P_{k}=-\overline{u_{i}^{\prime} u_{j}^{\prime}} \partial \bar{u}_{i} / \partial \bar{x}_{j}$ in the turbulence kinetic energy equation and has the correct order of magnitude providing the length scale $L_{T}$ and $L_{\epsilon}$ corresponds to the smallest resolved length scale in the flow field. Therefore, the first term on the right-hand side of (4) should not be damped. The second term on the right-hand side suggests that $F$ can be used to rescale the characteristic length scale $L_{T}$ in the turbulence viscosity relation. Note, Batten's et al.[5] idea of rescaling the turbulent viscosity is equivalently valid. A consistent FSM framework, which is consistent in the modeled length scale definitions, is obtained by reducing the damping of the Reynold-stress tensor to a damping of both characteristic length scales $L_{T}$ and $L_{\epsilon}$. Thus, the following length scale definition is used in the FSM framework $L_{h y b}=F \cdot L_{T}$ and $L_{T}=L_{\epsilon}$. It follows that $\nu_{T}=F C_{\mu} V_{T} L_{h y b}$ and $\epsilon=F^{-1} C_{\epsilon} V_{T}^{3} / L_{h y b}$. Using this approach the FSM turbulence-model contribution adjusts to the local and instantaneous state of the flow and the resulting damped Reynolds-stress tensor has to be considered as a subgrid-stress tensor and the turbulence kinetic energy $k$ and dissipation rate $\epsilon$ computed from the transport equations, turn into the subgrid quantities $k_{s g s}$ and $\epsilon_{s g s}$, respectively. It should be noted that for two-equation DES models, typically, only the length scale $L_{\epsilon}$ is modified and the length scale $L_{T}$ in the turbulence viscosity relation is left unchanged.

\subsection{Choice of underlying RANS model}

In order to investigate the sensitivity of results with respect to the underlying RANS model, the Flow Simulation Methodology is used in conjunction with two different RANS turbulence models of different levels of sophistication. 


\subsubsection{FSM- $k-\omega-S S T$}

The $k-\omega$-SST model[40,41] is chosen from the available range of two-equations models since it provides superior results to many other other two-equation models in a range of applications. The transport equations for the turbulent kinetic energy $k$ and the specific dissipation rate $\omega$ take the following form

$$
\begin{aligned}
& \frac{D k}{D t}=P_{k}-\epsilon+\frac{\partial}{\partial x_{j}}\left[\left(\nu+\sigma_{k} \nu_{T}\right) \frac{\partial k}{\partial x_{j}}\right], \\
& \frac{D \omega}{D t}=\frac{\gamma}{\nu_{T}} P_{k}-\beta \omega^{2}+\frac{\partial}{\partial x_{j}}\left[\left(\nu+\sigma_{\omega} \nu_{T}\right) \frac{\partial \omega}{\partial x_{j}}\right]+2\left(1-F_{1}\right) \frac{\sigma_{w 2}}{\omega} C D_{k \omega},
\end{aligned}
$$

where $P_{k}=-\overline{u_{i}^{\prime} u_{j}^{\prime}} \partial \bar{u}_{i} / \partial x_{j}$ is the production of turbulent kinetic energy and $C D_{k \omega}=\partial k / \partial x_{j} \partial \omega / \partial x_{j}$ is the turbulent cross-diffusion term, arising from the formal transformation of the equation for the dissipation rate $\epsilon$ to a form based on the specific dissipation rate $\omega$. The dissipation rate $\epsilon$ in the turbulent kinetic energy equation is modeled as $\epsilon=\beta^{*} k \omega$. The blending between the $\omega$-equation, which is used in the near-wall region, to the $\epsilon$-equation, which is used in the wake region of attached boundary layers, is achieved by the blending function $F_{1}$ and by blending the sets of closure constants. The turbulent viscosity is given by $\nu_{T}=a_{1} k / \max \left(a_{1} \omega, S F_{2}\right)$, where $S=\sqrt{S_{i j} S_{i j}}$ and $F_{2}$ is another blending function. The $k$ - $\omega$-SST model is used in conjunction with a linear stress-strain relationship.

Within the hybrid FSM approach the turbulence kinetic energy dissipation rate is given by

$$
\epsilon=F^{-1} \beta^{*} k \omega
$$

and the turbulence viscosity is obtained as

$$
\nu_{T}=F \frac{a_{1} k}{\max \left(a_{1} \omega, S F_{2}\right)} .
$$

Note that this formulation is equivalent to replacing the characteristic turbulence length scale in the definition of the dissipation rate and in the turbulence viscosity, with $L_{h y b}=F L_{T}=F L_{\epsilon}$ and $L_{T}=k^{1 / 2} / \beta^{*} \omega$.

\subsubsection{FSM-k- $\omega-E A S M$}

The EASM used in this study is a high-Reynolds-number formulation, since neither the transport equations nor the quasi-homogeneous pressure-strain 
model used in the EASM includes modifications to account for various effects the wall exerts on the flow. The Reynolds-stress tensor $\overline{u_{i}^{\prime} u_{j}^{\prime}}$ of the EASM formulation constitutes a non-linear stress-strain relationship, which can be written as $\overline{u_{i}^{\prime} u_{j}^{\prime}}=(2 / 3) k \delta_{i j}+k a_{i j}$, where the anisotropy tensor is given by

$$
a_{i j}=-2\left(\nu_{T} / k\right) S_{i j}^{*}+a_{i j}^{e x}
$$

with the strain rate tensor $S_{i j}^{*}=(1 / 2)\left(\partial \bar{u}_{i} / \partial x_{j}+\partial \bar{u}_{j} / \partial x_{i}\right)$ and the extraanisotropy tensor $a_{i j}^{e x}$, which introduces anisotropy in addition to the anisotropy resulting form the strain rate $S_{i j}^{*}$. For a vanishing extra-anisotropy tensor $a_{i j}^{e x}=0$, the stress-strain relationship reduces to the linear Boussinesq approximation.

The assumption of two-dimensional mean flow is invoked for the derivation of $a_{i j}^{e x}$. This assumption considerably reduces the complexity of the EASM and only adds moderate additional numerical expense compared to a linear two-equation model. It was shown in Weinmann \& Sandberg[42] that the twodimensional EASM formulation provides very similar results in complex threedimensional flows when compared to the more complex three-dimensional EASM formulation. In the limit of two-dimensional mean flow the extra anisotropy tensor can be written as

$$
a_{i j}^{e x}=\beta_{2}\left(S_{i k} S_{k j}-\frac{1}{3} I I_{S} \delta_{i j}\right)+\beta_{4}\left(S_{i k} \Omega_{k j}-\Omega_{i k} S_{k j}\right),
$$

with the invariants $I I_{S}=\operatorname{tr}\left\{S_{i k} S_{k j}\right\}, I I_{\Omega}=\operatorname{tr}\left\{\Omega_{i k} \Omega_{k j}\right\}$ and the strain and rotation-rate tensors $S_{i j}$ and $\Omega_{i j}$ which are normalized by the turbulent time scale $\tau=1 /\left(\beta^{*} \omega\right)$.

The detailed derivation of an explicit solution for a general quasi-linear AlgebraicRSM can be found in Wallin \& Johansen [43] and is not repeated here. The solution is obtained in form of the $\beta_{i}$ coefficients which, for two-dimensional mean flow, are given as

$$
\beta_{1}=-A_{1} N / Q, \quad \beta_{2}=2 A_{1} A_{2} / Q, \quad \beta_{4}=-A_{1} / Q
$$

where

$$
Q=N^{2}-2 I I_{\Omega}-\frac{2}{3} A_{2}^{2} I I_{S}
$$

In the present study the EASM is based on the pressure-strain model of Speziale et al. [44] which results in the coefficients

$$
A_{1}=1.22, \quad A_{2}=0.47, \quad A_{3}=0.88, \quad A_{4}=2.37 .
$$


The variable $N$ appearing in equation (11)-(12) corresponds to the ratio of production to dissipation and is governed by a non-linear relation. For twodimensional flows the non-linear equation has an explicit solution which is given by

$$
N=\left\{\begin{array}{l}
\frac{A_{3}}{3}+\left(P_{1}+\sqrt{P_{2}}\right)^{1 / 3}+\left(P_{1}-\sqrt{P_{2}}\right)^{1 / 3}, P_{2} \geq 0 \\
\frac{A_{3}}{3}+2\left(P_{1}^{2}-P_{2}\right)^{1 / 6} \cos \left[\frac{1}{3} \arccos \left(\frac{P_{1}}{\sqrt{P_{1}^{2}-P_{2}}}\right)\right], P_{2}<0
\end{array}\right.
$$

with

$$
\begin{aligned}
& P_{1}=\left[\frac{A_{3}^{2}}{27}+\left(\frac{A_{1} A_{4}}{6}-\frac{2}{9} A_{2}^{2}\right) I I_{S}-\frac{2}{3} I I_{\Omega}\right] A_{3}, \\
& P_{2}=P_{1}^{2}-\left[\frac{A_{3}^{2}}{9}+\left(\frac{A_{1} A_{4}}{6}+\frac{2}{9} A_{2}^{2}\right) I I_{S}+\frac{2}{3} I I_{\Omega}\right]^{3} .
\end{aligned}
$$

The turbulence viscosity is now a function of $\beta_{1}$ and takes the form

$$
\nu_{T}=\frac{-0.5 \beta_{1}}{\beta^{*}} \frac{k}{\omega}
$$

The relations above must be supplemented by a transport equation for the turbulent velocity scale and a transport equation for the turbulent length scale. The transport equations for the turbulent kinetic energy $k$ is equivalent to (5) and the specific dissipation rate $\omega$ for the EASM follows the proposal of Hellsten[45] and constitutes a recalibrated version of Menter's blending approach.

$$
\frac{D \omega}{D t}=\gamma \frac{\omega}{k} P_{k}-\beta \omega^{2}+\frac{\partial}{\partial x_{j}}\left[\left(\nu+\sigma_{\omega} \nu_{T}\right) \frac{\partial \omega}{\partial x_{j}}\right]+\frac{\sigma_{d}}{\omega} \max \left(C D_{k \omega}, 0\right)
$$

Within the hybrid FSM approach the dissipation rate is given by $\epsilon=F^{-1} \beta^{*} k \omega$, and the turbulence viscosity is $\nu_{T}=F\left(-0.5 \beta_{1} k \tau\right)$. The non-linear stress-strain relationship of the EASM model $\overline{u_{i}^{\prime} u_{j}^{\prime}}=(2 / 3) k \delta_{i j}+k a_{i j}$, requires that the damping function is applied to the extra-anisotropy tensor according to

$$
a_{i j}=-2\left(\nu_{T} / k\right) S_{i j}^{*}+F \cdot a_{i j}^{e x} .
$$

From the authors' point of view, the EASM model provides some interesting capabilities when employed in the context of a hybrid RAN/LES model. In addition to providing a more realistic representation of the subgrid stress and anisotropy field, the EASM model also introduces a dynamic response of the 
model to the resolved flow field by employing coefficients which depend on the strain and rotation rate invariants. For example, in stationary homogeneous isotropic turbulence, the EASM model dynamically reduced the constant $C_{\mu}=$ $-1 / 2 \beta_{1}$ to a mean value of $C_{\mu} \approx 0.028$ which is lower than the generally accepted value of $C_{\mu}=0.09$ in the RANS framework.

\subsection{A tailored hybrid convection scheme}

In LES applications, the subgrid-scale (SGS) model has to provide an adequate amount of dissipation, such that the turbulence energy cascade is correctly reproduced and no energy accumulates at the smallest resolved scales. The amount of dissipation provided by the SGS model may become very small with the consequence that any elevated level of numerical dissipation will inevitably contaminate the resolved flow field. Therefore, higher-order central schemes (CDS) with low levels of numerical dissipation are the preferred choice in LES. However, the unboundedness of CDS may result in spurious oscillations if the stability constraints are not satisfied[46]. In general, higher-order upwind or TVD schemes are not recommended for turbulence resolving simulations, since the additional numerical dissipation introduced by these schemes is significant, and will contaminate the resolved flow field. In RANS applications, the source terms and high-order nonlinearities present in the transport equations for turbulence quantities require more robust, yet less accurate, upwind or TVD schemes to ensure numerical stability. The increased levels of numerical dissipation induced by the second-order upwind or TVD schemes is usually smaller than the large diffusivity provided by the RANS model and hence, will not affect the predictions as much as in the case of LES.

Therefore, any unified RANS/LES approach will benefit from a tailored convection scheme which satisfies the opposed requirements RANS and LES place on the numerical scheme. A hybrid convection scheme tailored to Detached Eddy Simulation has been presented by Travin et al. [47]. A tailored secondorder accurate hybrid convection scheme for the FSM approach is obtained by a linear combination of the central-difference scheme (CDS) and a secondorder upwind scheme, using a flow-dependent flux-blending function. The face fluxes of the hybrid convection scheme are obtained from a linear combination of the form

$$
\phi_{f}=\left(1-\sigma_{b}\right) \phi_{f, C D S}+\sigma_{b} \phi_{f, L U D}
$$

where $\sigma_{b}$ is a flow-dependent blending function. The blending function is designed such that $\sigma_{b}=1$ if FSM operates in RANS mode $(F=1)$ and rapidly drops to zero if the damping function is $F<1$. The following function provides 
the desired behaviour

$$
\sigma_{b}=\tanh \left(A^{3}\right), \quad A=\max \left[f_{\eta} f_{\Delta} f_{z}-0.6,0\right] .
$$

Figure 1 shows instantaneous contours of the blending function $\sigma_{b}$ and the hybrid scheme of Travin et al. [47] for the flow around the two inline tandem cylinders. It is obvious that both schemes ensure that the irrotational freestream and boundary layers on both cylinder are computed using the upwinddifference scheme $\left(\sigma_{b}=1\right)$. In the wake of the first and second cylinder, $\sigma_{b}$ drops sharply to zero and the resolved flow is computed using the centraldifference scheme. It should be noted that using this hybrid convection scheme a stable solution was obtained, whereas no stable solution could be obtained for the tandem cylinder case if the central-difference scheme was employed everywhere in the domain.



(a) FSM scheme

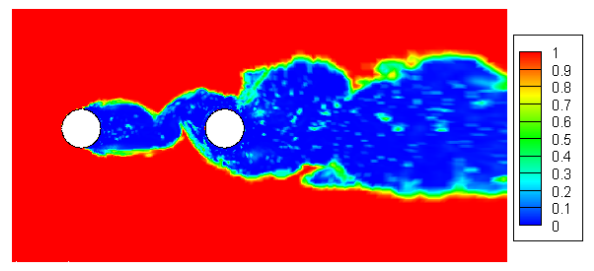

(b) Hybrid scheme of Travin et al. [47]

Fig. 1. Flux blending function $\sigma_{b}$ for the tandem cylinder configuration.

\subsection{Calibration}

Stationary homogeneous isotropic turbulence is used to calibrate and validate the LES capabilities of the modified FSM approach. This is achieved by determining suitable values for the free parameters $C_{\alpha}, \beta$ and $n$, which appear in the damping function $F$. In the calibration process, the total turbulence kinetic energy dissipation rate $\epsilon=\epsilon_{\text {sgs }}+\epsilon_{\nu}+\epsilon_{\text {num }}$, resulting form the combined effect of turbulence model formulation, viscosity and numerical dissipation, is optimised to achieve a physically consistent energy cascade. The calibration is performed in conjunction with the hybrid FSM convection scheme presented in section 2.3. The correct implementation of the IDDES and SAS models, which are used as reference, have also been validated using this approach.

To perform the calibration, simulations are performed in a cubic box with a side length of $L=2 \pi[\mathrm{m}], 64$ cells in each direction of the box and with periodic boundary conditions in all directions. The time step is set such that the CFL number remains below $C F L<0.4$. In order to circumvent the natural decay of isotropic turbulence a forcing of the momentum equations at low wavenumber is employed. The constant $C_{\alpha}$ is calibrated on a Taylor-scale Reynolds number 
of $R e_{\lambda}=732$, which is also studied by Kaneda [48] using DNS, and their results are used as a reference. The resulting energy spectra ,see figure 2 are averaged over more than 30 samples, which are sufficiently separated in time to ensure that they are not correlated. The optimized constants $C_{\alpha}$ take the value $C_{\alpha}=0.85$ for the FSM-SST and $C_{\alpha}=2.1$ for the FSM-EASM model.

The calibration has also been performed on a grid resolution of 32 cells in each direction. It is noted that the resulting model constants are slightly higher compared to the values presented for the resolution of 64 cells in each direction. The calibration has also been performed for a parallel channel flow, where FSM operates as a wall-modelled LES approach $\left(f_{z}=1\right)$. It was found that the results are very sensitive to the near-wall distribution of the damping function $F$ and that the sensitivity to the calibration constants is very small. Full details of the calibration simulations and procedure are given in Weinmann[49].

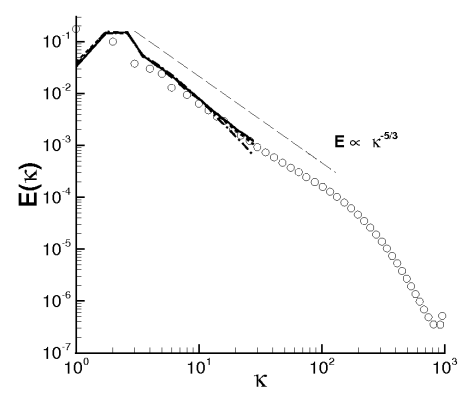

(a)

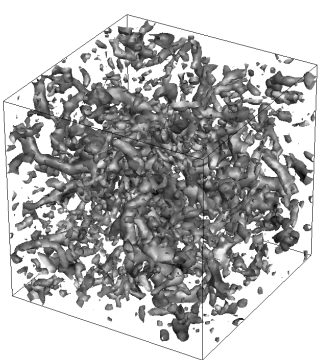

(b)

Fig. 2. Energy spectra $E(\kappa)$ and iso-contours of $Q=1 / 2\left(\Omega_{i j}^{*} \Omega_{i j}^{*}-S_{i j}^{*} S_{i j}^{*}\right)=3\left(1 / s^{2}\right)$. Symbols correspond to DNS[48]. (-) FSM-SST, (- - ) FSM-EASM, ( . . IDDES , (- - -) SAS.

The constants $\beta$ and $n$ are calibrated such that the FSM contribution function reduces to $F=0$ if the grid resolution is sufficient to resolve all scales of motion, i.e. when the ratio of grid resolution and Kolmogorov length scale are approximately unity $\left(\Delta / L_{\eta}=1\right)$. The calibration is performed for $R e_{\lambda}=35$ where the present grid is able to resolve all scales of motion. For the values $\beta=0.75$ and $n=1$ the correct DNS limit of the contribution function $F$ is achieved. Since the simulations in this work are performed on coarse grids, the function $f_{\eta}$ will remain inactive. In practice, $f_{\eta}$ will only become active when the flow is locally well resolved.

The value of the calibration constant $C_{\alpha}$ of the FSM-EASM model is significantly larger compared to the value for the FSM-SST model. This indicates that the two underlying RANS models predict different levels of turbulence length and velocity scales and that the FSM-EASM model is significantly less dissipative than the FSM-SST. The difference in the dissipative nature is explained by the sensitivity of the parameter $\beta_{1}$ in the turbulence viscosity 
Table 1

Simulated microphone positions (with respect to the centre of the upstream cylinder).

\begin{tabular}{ccc}
\hline \hline Name & $x / D$ & $y / D$ \\
\hline Microphone A & -8.33 & 27.817 \\
Microphone B & 9.11 & 32.49 \\
Microphone C & 26.55 & 27.815 \\
\hline \hline
\end{tabular}

relation of the EASM model to strain and rotation rate. In the present case, the constant $C_{\mu}=-0.5 \beta_{1}$ of the FSM-EASM reduces to $\left\langle C_{\mu}\right\rangle=0.028$, where the brackets \langle\rangle denote averaging in time and space. This value is significantly smaller compared to the generally accepted value of $C_{\mu}=0.09$ (away from solid walls) and hence requires a larger value for $C_{\alpha}$.

\subsection{Acoustic Method}

Acoustic pressures were computed using the unsteady flow data generated during flow simulation, for each turbulence model. An acoustic analogy approach was used, based on the theory of Curle[50]. Assuming a low Mach number subsonic flow, the sound radiated by a rigid, stationary body in a fluctuating flow can be written as the gradient of a surface pressure integral

$$
4 \pi c_{0}^{2}\left(\rho(\tilde{x}, t)-\rho_{0}\right)=\frac{\partial}{\partial x_{i}} \iint_{S} \frac{l_{j}}{r}\left[p \delta_{i j}\right] d S(\tilde{y})
$$

where $c_{0}$ and $\rho_{0}$ are the speed of sound and the fluid density in the medium at rest, respectively, and $\tilde{y}$ denotes a point on the rigid surface, separated from the observation point $\tilde{x}$ by the distance $r . l_{i}$ are the components of the unit vector that is normal to the surface. The square brackets denote a value taken at the retarded time $t-r / c_{0}$.

For ease of computation, the spatial derivative was converted to a temporal derivative using

$$
4 \pi c_{0}^{2}\left(\rho(\tilde{x}, t)-\rho_{0}\right)=\frac{1}{c_{0}} \iint_{S} \frac{x_{i}}{r^{2}} \frac{\partial}{\partial t} l_{i}\left[p \delta_{i j}\right] d S(\tilde{y})
$$

Equation 22 was evaluated for each computational test case to obtain a transient acoustic record at each microphone location used in the experimental study[20]. The locations of these microphones are listed in table 1. The positions of the microphones correspond to approximately one wavelength at the 
fundamental shedding tone, however, an analysis[27] shows near field effects are negligible for the frequency range of interest here.

Between $N_{S}=2257$ and 3348 hydrodynamic fields were stored for each case with sampling rates between $F_{S}=3126$ and $4168 \mathrm{~Hz}$, resulting in approximate maximum resolvable Strouhal numbers between $S t_{\max }=2.06$ and 2.74. Each acoustic record was converted to a power spectral density using a hamming window function on four data windows of equal length, resulting in a frequency resolution that varied between $\Delta f=3.24$ and $6.06 \mathrm{~Hz}$.

Data were collected at 13 spanwise locations on each cylinder and 40 individual transient pressure records were collected about the circumference at each spanwise location. The span of each cylinder in the experiment was $16 D$ but the simulation was performed for a span of $3 D$. To correct for the different span lengths, spanwise amplitude correction was performed using the method of Seo and Moon[51]. The simulated data were not shifted in frequency to match the main shedding tone of the experiments.

\subsection{Computational Details}

The tandem cylinder configuration considered in this work has a gap spacing of $x / D=3.7$ (from centre to centre). The Reynolds number is $R e_{D}=1.66 \times 10^{5}$ based on the cylinder diameter $D$ and free-stream velocity $U_{0}$. Experimental data from the QFF and BART facilities[20,25,23] are used to assess the performance of the hybrid RANS/LES methodologies introduced above.

All models to be tested here are implemented into the open source finite volume code OpenFOAM[52,53]. The simulations for the tandem cylinder configuration are performed using a segregated incompressible solver where the coupling between the pressure and velocity is achieved by the PISO algorithm. The convective fluxes in the flow equations are discretised using the hybrid

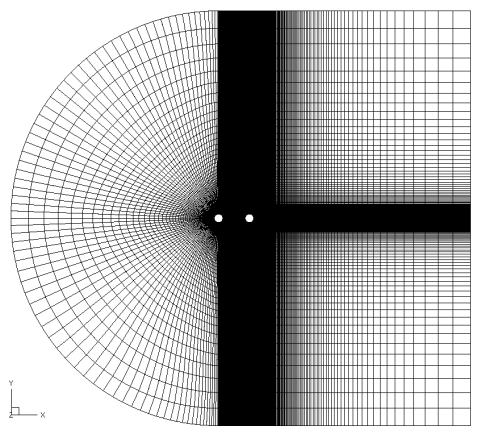

(a)

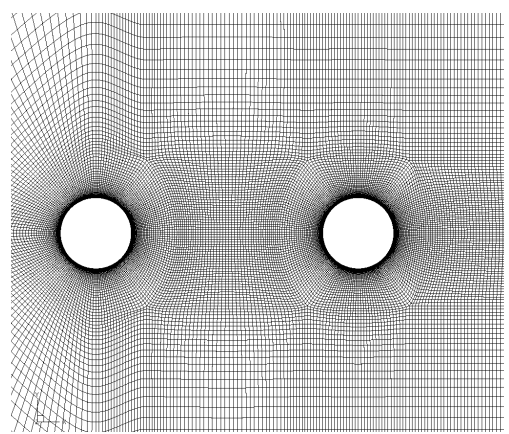

(b)

Fig. 3. Computational grid. 
FSM difference scheme and a second-order upwind scheme is used for the turbulence transport equations. The viscous terms use a second-order centraldifference scheme. Time stepping is performed using an implicit second-order backward-difference scheme. The time step is set to $0.003 \mathrm{D} / U_{0}$ such that the CFL number does not exceed 0.5.

The computational setup consists of a three-dimensional C-type domain with a spanwise extend of $z / D=3$. The upstream boundary is placed $25 D$ away from the cylinders and the outflow boundary is located at $x / D=30$ downstream of the centre of the first cylinder. Periodic boundary conditions are used in the spanwise direction, and zero-gradient boundary conditions on the outflow boundary. Each two-dimensional plane $(x-y)$ (see figure 3) is discretized with approximately 52,000 cells using an average first wall-normal grid spacing, on both cylinders, of around $y_{1}^{+}=0.7$ and a maximum value of $y_{1}^{+}=1.7$. The number of cells in the spanwise direction is 40 , so as to achieve almost isotropic cells in the gap region between the cylinders. The total cell count is approximately $2 \times 10^{6}$ cells. The grid resolution used here and the total number of cells of the baseline grid is significantly less compared to what was used in other contributions to the BANC I workshop (typically $7-133 \times 10^{6}$ cells, see Lockard[34]). The turbulence kinetic energy and specific dissipation rate at the inflow are respectively prescribed as $k_{\infty}=10^{-6} U_{0}^{2}$ and $\omega_{\infty}=5 U_{0} / D$ [54] such that immediate transition occurs in the boundary layer.

\section{RESULTS}

\subsection{Averaging and Statistical Convergence}

For all simulations, the flow is allowed to develop from the initial flow field for approximately $115 \mathrm{D} / U_{0}$ time units. After the initial settling period, statistical quantities are computed by averaging over $700 D / U_{0}$ time units. For postprocessing purposes the time-averaged quantities are additionally averaged over the homogeneous spanwise direction.

In order to verify the statistical convergence, the distributions of mean-surfacepressure coefficient $C_{p}$ and fluctuating-surface-pressure coefficient $C_{p_{r m s}^{\prime}}$ are shown in figure 4 , for both cylinders, for averaging over $230 \mathrm{D} / U_{0}, 460 \mathrm{D} / U_{0}$ and $690 D / U_{0}$ time units. It is obvious that the mean-surface-pressure distribution is already converged after averaging over $230 \mathrm{D} / U_{0}$ time units. The fluctuatingsurface-pressure distribution requires time-averaging over at least $460 \mathrm{D} / U_{0}$ time units before adequately converged statistics are obtained. Averaging over $460 D / U_{0}$ time units is therefore deemed to be sufficient for obtaining statistical convergence. 


\subsection{Effect of Spanwise Domain Width}

The effect of the spanwise width of the computational domain is investigated by performing simulations on three computational grids with different spanwise extents. The first grid has a spanwise width of $3 D$ and 40 cells in the spanwise direction (FSM-3-40), the second grid has a spanwise width of $6 D$ and 80 cells in the spanwise direction (FSM-6-80) and the largest grid has a spanwise width of $12 D$ and 160 cells in the spanwise direction (FSM-12-160).

The distributions of mean-surface-pressure coefficient $C_{p}$ and fluctuating-surfacepressure coefficient $C_{p_{r m s}^{\prime}}$ are shown in figure 5, for the upstream and downstream cylinder. The mean-surface-pressure distributions on both cylinders are virtually indistinguishable and are therefore not sensitive to the spanwise domain width.

\subsection{Sensitivity to the Turbulence Model}

The sensitivity of results with respect to the underlying RANS model is investigated. For this purpose, simulations are performed using the FSM-SST

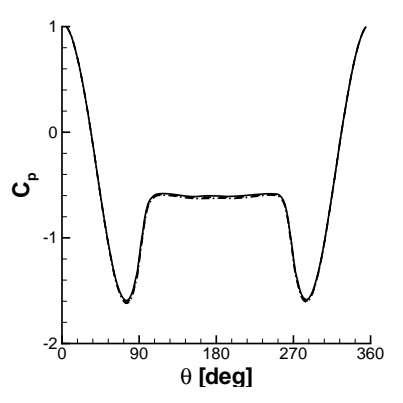

(a) $C_{p}$ front cylinder.

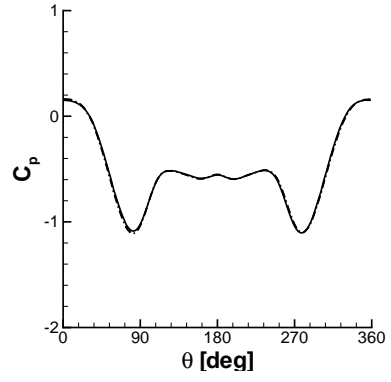

(b) $C_{p}$ rear cylinder.



(c) $C_{p_{r m s}^{\prime}}$ front cylinder.



(d) $C_{p_{r m s}^{\prime}}$ rear cylinder.

Fig. 4. Mean-surface-pressure $C_{p}$ and fluctuating-surface-pressure coefficient $C_{p_{r m s}^{\prime}}$. (-) $t=230 \mathrm{D} / U_{0},(--) t=460 \mathrm{D} / U_{0},(-\cdot-) t=690 \mathrm{D} / U_{0}$. 


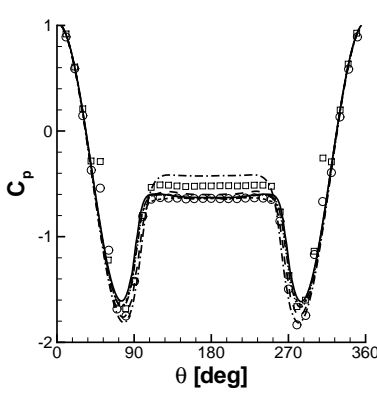

(a) $C_{p}$ front cylinder.



(b) $C_{p}$ rear cylinder.



(d) $C_{p_{r m s}^{\prime}}$ rear cylinder.

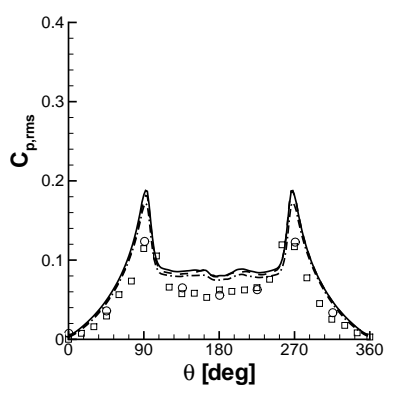

(c) $C_{p_{r m s}^{\prime}}$ front cylinder.

Fig. 5. Mean-surface-pressure $C_{p}$ and fluctuating-surface-pressure coefficient $C_{p_{r m s}^{\prime}}$. Front cylinder: (o) BART, ( $\square$ ) QFF. Rear Cylinder: (o) BART, ( $\square$ ) BART (rear cylinder tripped). (-) FSM-3-40, (- - ) FSM-6-80, (- - -) FSM-12-160.

and FSM-EASM formulations. In addition, results obtained by IDDES and SAS are also included in the comparison with the reference data. The IDDES and SAS are both based on the $k$ - $\omega$-SST model and use a second-order accurate variant of the hybrid convection scheme of Travin et al. [47]. The SAS employs the same definition of $\Delta$ as used for the FSM approach (cube root of the cell volume), whereas IDDES employs a more complex formulation which shows a linear increase close to the wall and approaches the maximum local grid spacing $\Delta=\max \left(\Delta_{x}, \Delta_{y}, \Delta_{z}\right)$ away from the wall. All simulations are performed on the baseline computational grid with a spanwise width of $3 D$ and 40 cells in the spanwise direction.

\section{Instantaneous Flow Field}

Instantaneous snapshots of the flow fields are visualised in figure 6 using isocontours of the second invariant of the velocity gradient tensor $Q=1 / 2\left(\Omega_{i j}^{*} \Omega_{i j}^{*}-S_{i j}^{*} S_{i j}^{*}\right)$. This quantity can be used to identify coherent or vorticity-dominated structures in the flow field. In addition, an snapshot of the instantaneous turbulence viscosity ratio $\nu_{T} / \nu$ at he upstream cylinder for the plane $z / D=1.5$ is shown in figure 8.

It is evident, that all hybrid RANS/LES methodologies show the presence of resolved turbulence structures in the flow field. The FSM-SST and IDDES 
model show the lowest level of turbulence viscosity in the shear layer on the upstream cylinder which allows for a more rapid growth of shear layer instability. The SAS model predicts a delayed onset of shear layer instability and the resolved shear layer predicted by the FSM-EASM model does not show much resemblance to the typical Kelvin-Helmholtz instability mechanisms. For both models the shear layer instability is suppressed by elevated levels turbulence model contribution. These observations are confirmed in figure 7 , which shows contour plots of instantaneous spanwise vorticity $\omega_{z} D / U_{0}$ obtained from the simulations and PIV data from the experiment.

A possible cause of the high levels of turbulence viscosity in the free shear layer predicted by the FSM-EASM is the dynamic response of the turbulence viscosity to the local strain and rotation-rate through $C_{\mu}=-0.5 \beta_{1}$. For the calibration case of resolved isotropic turbulence $C_{\mu}$ reduced to $C_{\mu}=0.028$. As a consequence, the calibration constant $C_{\alpha}$ of the FSM-EASM approach was found to be significantly higher compared to the FSM-SST. On the other hand, in the free shear layer on the upstream cylinder a value of $C_{\mu} \approx 0.05$ is predicted. The combination of the large value for $C_{\alpha}$ and the larger value for $C_{\mu}$ in the free shear layer may result in a too high turbulence viscosity and an overly large damping of the shear layer instability. It is also evident from figure 8 that the turbulence viscosity produced by the FSM-EASM just behind the upstream cylinder is excessively large. This stems from a weakness of the EASM formulation which produces overly large values for $C_{\mu}$ when the strain-rate invariant $I I_{S}$ becomes small or vanishes.

The resolved flow fields also show the presence of streamwise vortices in the gap region, with a characteristic spanwise extent of 2-3 cell widths $\Delta_{z}$. These structures are less pronounced in the predictions of the FSM-EASM model, which exhibits more chaotic, irregular vortical structures in the flow field. The IDDES and SAS turbulence models show generally larger vortical structures compared to the FSM approaches. The predictions of the flow field in the wake of the downstream cylinder is similarly resolved by all turbulence models. Only the FSM-EASM is able to maintain turbulence structures much farther downstream of the rear cylinder. This is associated with the variable constant $C_{\mu}$ in the eddy-viscosity relation, which reduces to $C_{\mu} \approx 0.04-0.05$ for the EASM model. 


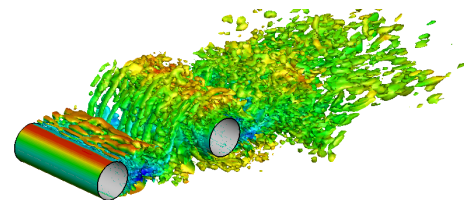

(a) FSM-SST



(c) IDDES



(b) FSM-EASM

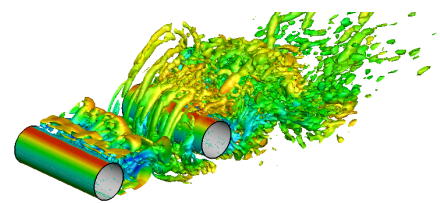

(d) SAS

Fig. 6. Iso-contours of $Q=1.5 U_{0}^{2} / D^{2}$, coloured by the instantaneous streamwise velocity.

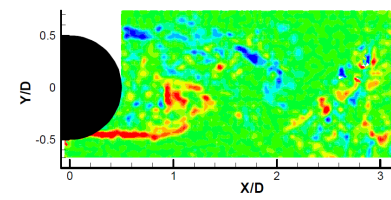

(a) Experiment [25]

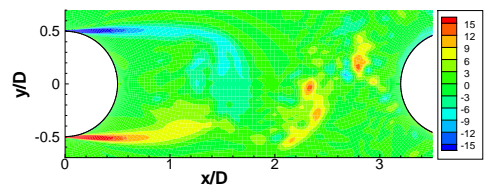

(c) FSM-EASM



(b) FSM-SST

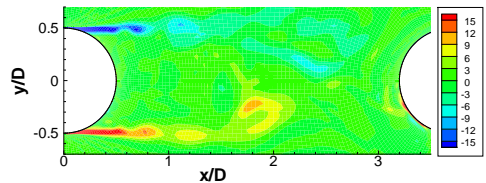

(d) IDDES



(e) SAS

Fig. 7. Normalized instantaneous spanwise vorticity $\omega_{z} D / U_{0}$.

\section{Alternative Mean Flow Field}

In has been mentioned in the introduction to this test case, that the distance between the cylinders sets the type of interaction. Suppression of vortex shedding from the upstream cylinder is usually observed for smaller gap spacings than used here. However, according to Lockard [34], some experiments conducted with the same gap spacing of $3.7 D$ at different Reynolds number and spanwise lengths of the cylinders have also shown an alternative state with suppressed vortex shedding from the upstream cylinder. It was found that IDDES converges to the first state with vortex shedding from the upstream cylinder for approximately $380 D / U_{0}$ time units. After a transition phase and a simulation time larger than $500 D / U_{0}$ time units, IDDES converges to a 


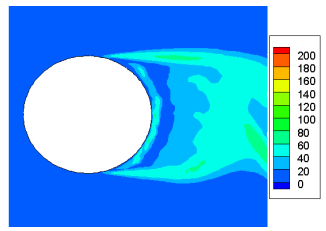

(a) FSM-SST

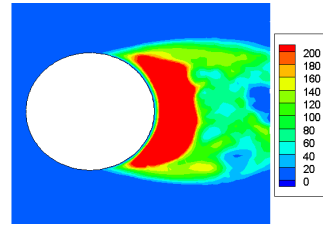

(b) FSM-EASM



(c) IDDES

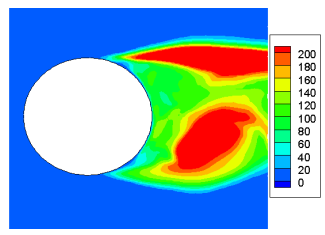

(d) SAS

Fig. 8. Instantaneous turbulence viscosity ratio $\nu_{T} / \nu$ at the upstream cylinder for the plane $z / D=1.5$.

second state, with suppressed vortex shedding from the upstream cylinder. The second state has also been observed in a simulation using the FSM-SST model on a grid with finer spanwise resolution, and has also been reported by some participants of the BANC I workshop [34]. It is not entirely clear what causes the transition to the alternative state but, it is expected, that a range of parameters such as computational grid, turbulence model and numerical discretisation contribute to this effect.

\section{Lift and Drag}

The predicted drag coefficient $C_{D}=F_{x} /\left(0.5 \rho U_{0} A\right)$ is summarised in table 2 , together with the primary vortex-shedding frequency and rms-values of fluctuating lift and drag coefficients. The primary vortex-shedding frequency measured in the experiment is $178 \mathrm{~Hz}$, which corresponds to a Strouhal number of $S t=0.231$. The FSM-SST, FSM-EASM and SAS models are able to accurately predict the primary-vortex shedding frequency observed in the experiment. IDDES predicts a Strouhal number that is too high, $S t=0.279$. In addition, the drag coefficient $C_{D}$, on the upstream cylinder as well as the $C_{L_{r m s}^{\prime}}$, are too small on both cylinders compared to the predictions of the other models. Note that the IDDES results are evaluated for the first $380 \mathrm{D} / U_{0}$ time units only where the vortex-shedding from the upstream cylinder is still present in the simulation.

The differences observed for the IDDES model can possibly be explained by the location of the separation point on the first cylinder. The IDDES model predicts the separation point of the mean flow at $\theta \approx 102^{\circ}, 258^{\circ}$, whereas, for example, the FSM-EASM predicts earlier separation at $\theta \approx 96^{\circ}, 264^{\circ}$. Due to the delayed separation predicted by the IDDES model, the development of the wake exhibits the behaviour of a wake at an effectively higher Reynolds number, which, according to Schewe[55] shows a similar trend to what is ob- 
Table 2

\begin{tabular}{c|c|c|c|c|c|c|c}
\hline \multicolumn{2}{l|}{} & \multicolumn{3}{c|}{ Upstream cylinder } & \multicolumn{3}{c}{ Downstream cylinder } \\
\hline Model & St & $C_{D}$ & $C_{L_{r m s}^{\prime}}$ & $C_{D_{r m s}^{\prime}}$ & $C_{D}$ & $C_{L_{r m s}^{\prime}}$ & $C_{D_{r m s}^{\prime}}$ \\
\hline \hline FSM-SST & 0.228 & 0.616 & 0.139 & 0.029 & 0.419 & 0.695 & 0.098 \\
\hline FSM-EASM & 0.234 & 0.626 & 0.148 & 0.022 & 0.383 & 0.695 & 0.098 \\
\hline IDDES & 0.279 & 0.397 & 0.036 & 0.017 & 0.409 & 0.425 & 0.059 \\
\hline SAS & 0.228 & 0.614 & 0.171 & 0.040 & 0.415 & 0.665 & 0.101 \\
\hline
\end{tabular}

Lift and drag coefficients.

served here, namely an increase in vortex-shedding frequency and a reduction in drag coefficient.

A possible explanation for the delayed separation observed with the IDDES is the ability of the model to elevate the turbulence model contribution over that of the underlying RANS model (through the function $f_{e}$ ). This function is intended to compensate the excessive drop in turbulence viscosity near the switching location from RANS to LES. In the present simulations, the function $f_{e}$ is active inside the boundary layer upstream of the separation point. Thus, the elevated level of turbulence model contribution shifts the separation point slightly downstream. This behavior has also been observed independently by Xiao et al. [56] who investigated the performance of DDES and IDDES for the tandem cylinder flow. The separation point of IDDES was found slightly downstream compared to DDES and the primary vortex shedding frequency is increased to $S t=0.272$.

\section{Mean Flow Field}

The distributions of the mean-surface-pressure coefficient $C_{p}$ and fluctuatingsurface-pressure coefficient $C_{p_{r m s}^{\prime}}$ are shown in figure 9 . All turbulence models are able to predict the surface-pressure coefficient in good agreement with the reference data. The IDDES model shows the most pronounced suction peaks at $\theta \approx 90^{\circ}$ and $\theta \approx 270^{\circ}$ and over-predicts the base pressure in the recirculation region of both cylinders. On the upstream cylinder, all other turbulence models reproduce the base pressure level observed in the BART facility, and predict a magnitude of the suction peaks, which matches the data from the QFF facility. On the downstream cylinder, the simulations are in good agreement with the reference data, and reproduce the measurement of the BART facility, which included a boundary-layer trip on the downstream cylinder.

The predictions of fluctuating-surface-pressure coefficient on the upstream cylinder reflects the predictions of the rms-values of lift and drag coefficient discussed above. The IDDES model, which showed the lowest values of $C_{L_{r m s}^{\prime}}$ and $C_{D_{r m s}^{\prime}}$, also consistently under-predict the surface-pressure fluctuation lev- 
els $C_{p_{r m s}^{\prime}}$. In addition, the location of the peak values, which approximately correspond to the separation location, are too far downstream and confirm the delayed separation. Similarly, the SAS model shows the highest levels of $C_{L_{r m s}^{\prime}}$ and $C_{D_{r m s}^{\prime}}$ and hence significantly over-predicts the intensity of surfacepressure fluctuations. All FSM models predict the same results of $C_{p_{r m s}^{\prime}}$ in the separated flow region and predict the magnitude to be slightly too high compared with the reference data.

The predictions of mean streamwise velocity on the centerline, $y / D=0$ are shown in figure 10 . The velocity in the gap region clearly show a large sensitivity to the turbulence model. The FSM-SST model predicts the velocity distribution in excellent agreement with reference data. The FSM-EASM, which is based on more sophisticated RANS model, and SAS both show a too small recirculation zone, whereas the IDDES model predicts a too large recirculation zone behind the upstream cylinder. Concerning the velocity distribution downstream of the rear cylinder, FSM-SST, IDDES and SAS provide similar predictions in excellent agreement with the BART reference data where the boundary-layer is tripped on the downstream cylinder. The FSM-EASM model over-predict the extent of the recirculation region behind the rear cylinder. This is associated with the mean separation point being slightly too far upstream, which consequently changes the trajectory of the free-shear layer and hence results in the too large recirculation zone.

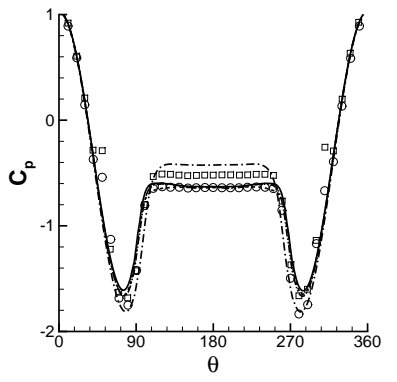

(a) $C_{p}$ front cylinder.

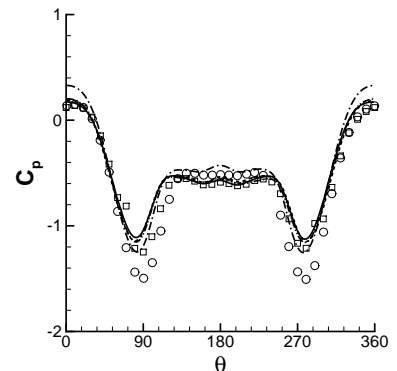

(b) $C_{p}$ rear cylinder.

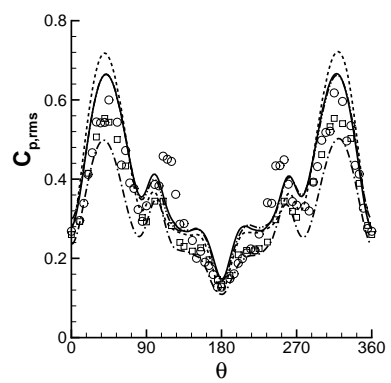

(d) $C_{p_{r m s}^{\prime}}$ rear cylinder.

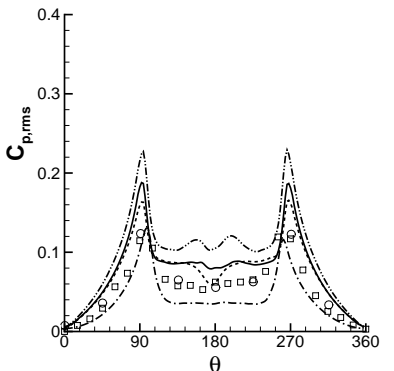

(c) $C_{p_{r m s}^{\prime}}$ front cylinder.

Fig. 9. Mean-surface-pressure $C_{p}$ and fluctuating-surface-pressure coefficient $C_{p_{r m s}^{\prime}}$. Front cylinder: (o) BART, ( $\square$ ) QFF. Rear Cylinder: (o) BART, ( $\square$ ) BART (rear cylinder tripped). (-) FSM-SST, (- -) FSM-EASM, (-- -) IDDES, (-*-) SAS. 




(a) $U / U_{0}$ gap region.

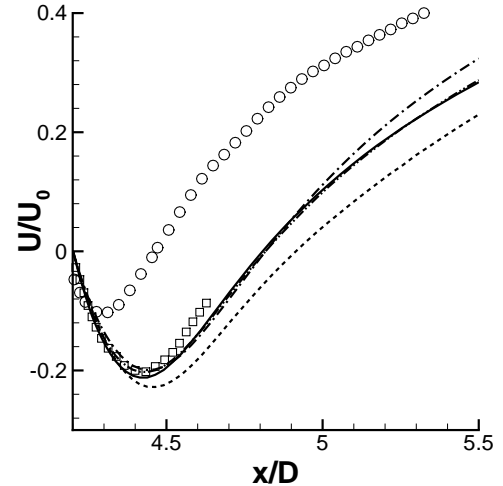

(b) $U / U_{0}$ aft of rear cylinder.

Fig. 10. Streamwise velocity profiles $U / U_{0}$ on the centreline $(y / D=0)$. (o) BART, $(\square)$ BART (rear cylinder tripped). (-) FSM-SST, (- - ) FSM-EASM, (- - -) IDDES, (- - - ) SAS.

Contour plots of turbulence intensity $I_{x}=\bar{u}_{r m s}^{\prime} / U_{0}$ and $I_{y}={\overline{v^{\prime}}}_{r m s} / U_{0}$ are shown in figures 11 and 12. The resolved turbulence kinetic energy in the gap region is predicted very similar by the hybrid RANS/LES turbulence models. Only the IDDES model predicts much lower levels of resolved turbulence kinetic energy throughout the gap region. The low intensity of velocity fluctuations results in less efficient entrainment of high-speed fluid from the free-stream, which contributes to the over-prediction of the size of the recirculation zone behind the upstream cylinder. All other turbulence models predict a higher intensity of velocity fluctuations, which enhances the entraining of fluid from the free-stream and consequently adds to the shortening of the recirculation zone behind the upstream cylinder.

Predictions of spanwise surface-pressure correlation $R_{p p}$ are shown in figure 13 . The IDDES model allows the spanwise surface-pressure correlation to drop to approximately $R_{p p} \approx 0.7$ on the first cylinder, and hence provides good agreement with the reference. In the predictions of all other cases, the spanwise correlation remains too high $R_{p p}>0.8$, compared to the corresponding measurements. The FSM-EASM shows overall the highest level of correlation $R_{p p}$. The high levels of correlation is also reflected in the iso-contour plots of $Q$ in figure 6 , where the free-shear layer shows a strong two dimensionality with almost no variation in the spanwise direction. On the downstream cylinder, the IDDES model again shows a rapid drop of $R_{p p}$ and reaching levels much below the corresponding measurements. The best agreement is achieved by the SAS model, which follows the trend observed in the experiment. The results of the FSM-SST model is of a similar quality, however, the correlation $R_{p p}$ remains somewhat too high. The more advanced FSM-EASM formulation shows least agreement with the reference data, and the spanwise correlation remains significantly too high over the cylinder span. 


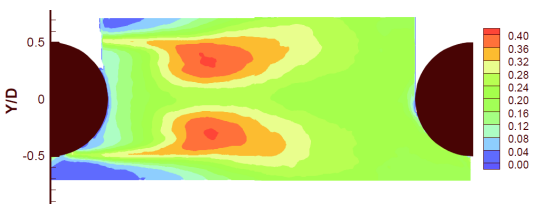

(a) Experiment.



(c) FSM-EASM.



(b) FSM-SST.

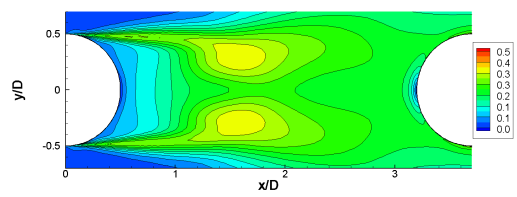

(d) IDDES.

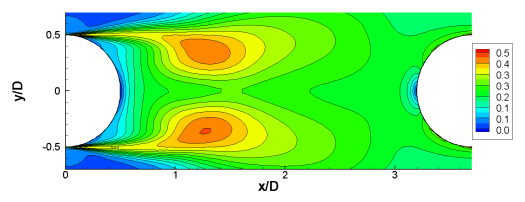

(e) SAS.

Fig. 11. Turbulence intensity $I_{x}={\overline{u^{\prime}}}_{r m s} / U_{0}$.

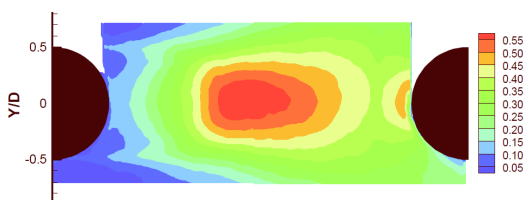

(a) Experiment.

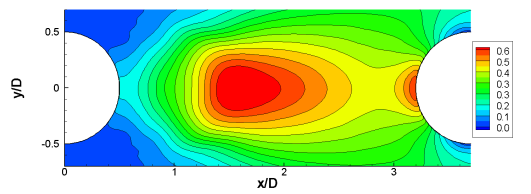

(c) FSM-EASM.

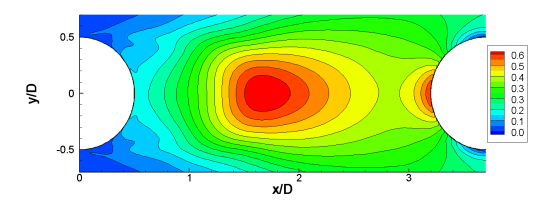

(b) FSM-SST.

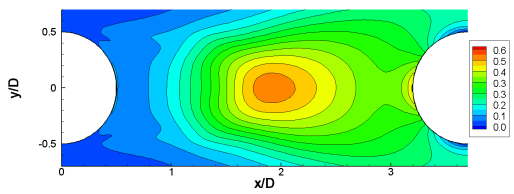

(d) IDDES.

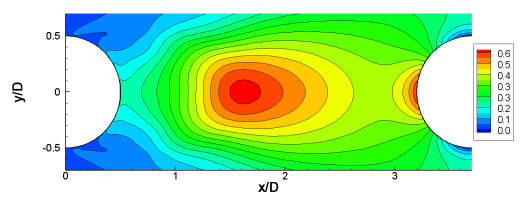

(e) SAS.

Fig. 12. Turbulence intensity $I_{y}={\overline{v^{\prime}}}_{r m s} / U_{0}$.

\subsection{Acoustic results}

Acoustic results for each turbulence model are shown for the three different Microphone positions in Figs. 14-16. In each case, experimental data[20] are compared with the simulated results. The FSM-SST turbulence model obtains excellent agreement below $S t \sim 1.2$ for all microphone positions. Above this 
value, the simulated broadband levels deviate from the experimental measurements. The deviation at higher Strouhal numbers reflects both the capacity of the turbulence model to properly resolve the smaller eddies and, possibly, the effect of acoustic scattering between the cylinders, which is not taken into account in the acoustic calculation.

The FSM-EASM model has somewhat similar performance to the FSM-SST model; however, the agreement is not as satisfactory. The IDDES model has difficulty capturing both the levels and the frequency of vortex shedding but has slightly better performance at higher Strouhal number. The SAS performs very well at low Strouhal number $(S t \lesssim 1)$ for microphones A and B, with some small degradation in the ability to resolve the harmonics at microphone C. The SAS model cannot reproduce the high frequency broadband levels observed in the experiment and this is attributed to the reasons given above.

\section{CONCLUSIONS}

A hybrid RANS/LES/DNS framework is presented, which seamlessly operates between RANS and DNS mode. This is achieved by a revised Flow Simulation Methodology, where the turbulence modelling contribution of a RANS model is rescaled using a damping function. The Flow Simulation Methodology is proposed in conjunction with a newly developed damping function and a tailored convection discretisation scheme, which further enhances the reliability and predictive accuracy of FSM. It is conjectured that a sophisticated RANS model will improve the overall quality of the predictions of any hybrid RANS/LES model, not only in the RANS region, but also in the LES region. For this reason, the FSM approach is presented and calibrated for the



(a) $R_{p p}$ at $\theta=135^{\circ}$ on front cylinder

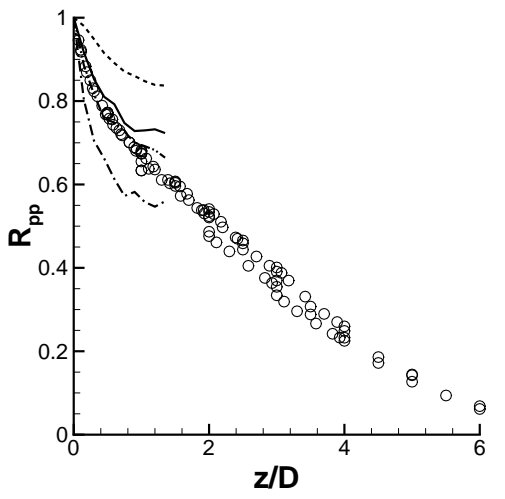

(b) $R_{p p}$ at $\theta=135^{\circ}$ on rear cylinder

Fig. 13. Spanwise surface-pressure correlation $R_{p p}$. (o) BART measurements. (-) FSM-SST, (- - ) FSM-EASM, (- - -) IDDES, $(-\cdots-)$ SAS. 


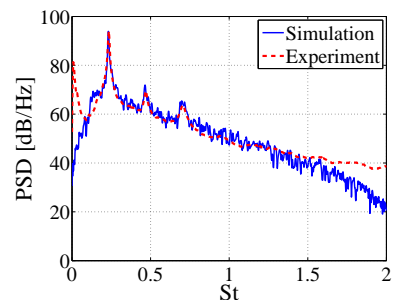

(a) FSM SST

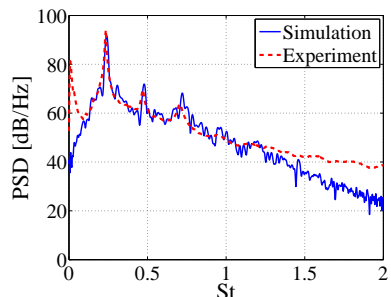

(b) FSM EASM

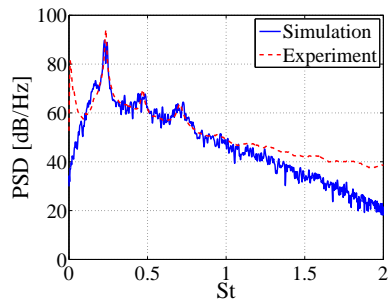

(d) SAS

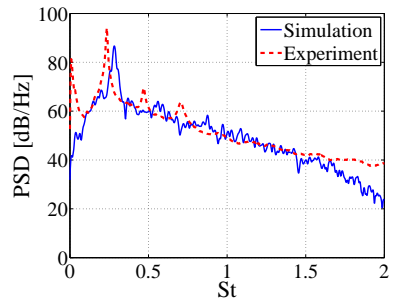

(c) IDDES

Fig. 14. Acoustic spectra comparison at Microphone A. The simulations are compared with NASA experimental data[20].

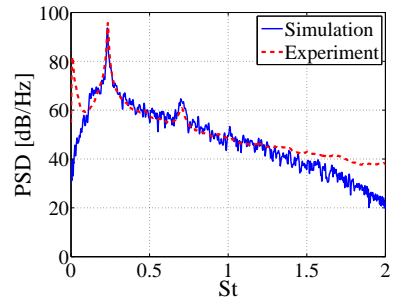

(a) FSM SST



(b) FSM EASM



(c) IDDES

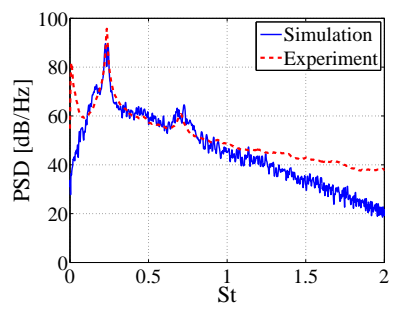

(d) SAS

Fig. 15. Acoustic spectra comparison at Microphone B. The simulations are compared with NASA experimental data[20].

$k$ - $\omega$-SST model and for the more sophisticated $k-\omega$-EASM model, which is based on a non-linear stress stain relationship and therefore better predicts anisotropy and non-equilibrium of the unresolved scales.

The flow around the tandem cylinder configuration is very sensitive to the turbulence model used. The IDDES model transitioned after long simulation times to an alternative mean-flow state, where the vortex shedding is suppressed from the upstream cylinder. The FSM-SST model was found to provide the best agreement of the hydrodynamic field with reference data on the 


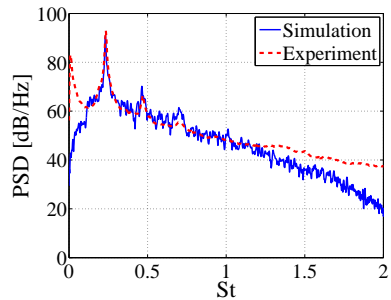

(a) FSM SST



(b) FSM EASM



(d) SAS

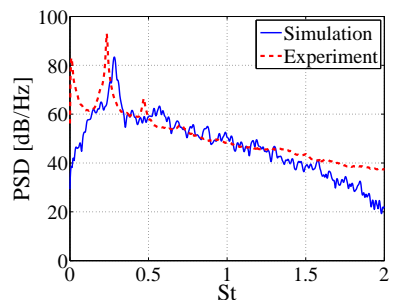

(c) IDDES

Fig. 16. Acoustic spectra comparison at Microphone C. The simulations are compared with NASA experimental data[20].

coarse computational grid followed by the FSM-EASM and SAS models. Despite the advanced modelling framework of the FSM-EASM, the predictions could not be improved over the FSM-SST. A possible cause is the sensitivity of the EASM formulation to local strain and rotation-rate, which is reflected in a large dynamic range of the modelling coefficients, depending on the flow under investigation. As a result, the FSM-EASM suppressed the free shear-layer instability on the upstream cylinder.

On the other hand, most results are insensitive to the spanwise domain width. This is linked to a spurious strong correlation of the flow in the spanwise direction. With increasing spanwise domain width, the intensity of surface-pressure fluctuations on the upstream cylinder reduces somewhat. Nevertheless, the difference is not pronounced enough to justify the increased computational cost associated with large spanwise domain widths.

The acoustic fields predicted, using an acoustic analogy based on Curle's theory, also show good agreement with experimental results. The quality of the acoustic results reflect the ability of the various turbulence models to recreate the turbulent flow fields, especially in the region between the two cylinders and importantly, the surface pressure distribution in the downstream cylinder. This correlation can be observed in the spanwise surface pressure correlation comparison data. Here, the best comparison with experimental data is obtained with the FSM-SST and SAS models, which is the same observed trend in the acoustic results. This suggests that the correct recreation of spanwise length scales is important for accurate acoustic predictions, especially in complex wake interaction cases such as the one presented in this paper. 


\section{ACKNOWLEDGMENTS}

The first author acknowledges financial support from the School of Engineering

Science at the University of Southampton.

\section{References}

[1] P. R. Spalart, W. H. Jou, M. Strelets, S. R. Allmaras, Comments on the Feasibility of LES for Wings, and on a Hybrid RANS/LES Approach, First AFOSR International Conference on DNS/LES, Ruston, Louisiana, USA.

[2] P. Sagaut, S. Deck, M. Terracol, Multiscale and Multiresolution Approaches in Turbulence, Imperial College Press, 2006.

[3] J. Froehlich, D. von Terzi, Hybrid LES/RANS Methods for the Simulation of Turbulent Flows, Progress in Aerospace Sciences 44, 5 (2008) 349-377.

[4] C. G. Speziale, A Combined Large-Eddy Simulation and Time-Dependent RANS Capability for High-Speed Compressible Flows, Journal of Scientific Computing 13, 3 .

[5] P. Batten, U. Goldberg, S. Chakravarthy, Interfacing Statistical Turbulence Closures with Large-eddy Simulation, AIAA Journal 42, 3.

[6] H. L. Zhang, C. R. Bachman, H. F. Fasel, Application of a New Methodology for Simulations of Complex Turbulent Flows, AIAA Paper 2000-2535.

[7] H. F. Fasel, J. Seidel, S. Wernz, A Methodology for Simulations of Complex Turbulent Flows, Journal of Fluids Engineering 124, 4 (2002) 933-942.

[8] P. Batten, U. Goldberg, S. Chakravarthy, Sub-Grid Turbulence Modelling for Unsteady Flow with Acoustic Resonances, AIAA Paper No. 00-0473.

[9] C. G. Speziale, Turbulence Modeling for Time-Dependent RANS and VLES: A Review, AIAA Journal 14, 3.

[10] H. F. Fasel, D. A. von Terzi, R. D. Sandberg, A Methodology for Simulating Compressible Turbulent Flows, Journal of Applied Mechanics 73, 3 (2006) 405412.

[11] F. R. Menter, M. Kuntz, R. Bender, A Scale-Adaptive Simulation Model for Turbulent Flow Predictions, AIAA 2003-0767, 41st Aerospace Sciences Meeting and Exhibit, Reno, Nevada.

[12] B. Chaouat, R. Schiestel, A new partiall integrated transport model for subgridscale stresses and dissipation rate for turbulent developing flows, Physics of Fluids 17. 
[13] R. Schiestel, A. Dejoan, Towards a new partially integrated transport model for coarse grid and unsteady turbulent flow simulations, Theoretical and Computational Fluid Dynamics 18 (2005) 443-468.

[14] C. Fureby, A. D. Gosman, G. Tabor, H. G. Weller, Large Eddy Simulation of Turbulent Channel Flows, in: Turbulent Shear Flows 11, 1997.

[15] C. Fureby, G. Tabor, H. G. Weller, A. D. Gosman, Differential subgrid stress models in large eddy simulation, Physics of Fluids 9, 11 (1997) 3578-3580.

[16] L. Marstropp, G. Brethouwer, O. Grundestam, A. V. Johanson, Explicit algebraic subgrid stress models with application to rotating channel flow, Journal of Fluid Mechanics 639 (2009) 403-432.

[17] N. Ashton, A. Revell, R. Prosser, J. Uribe, Development of an alternative delayed detached-eddy simulation formulation based on elliptic relaxation, AIAA journal 51 (2) (2012) 513-519.

[18] M. Zdravkovich, Review of flow interference between two circular cylinders in various arrangements, ASME Journal of Fluids Engineering 99 (1987) 618-631.

[19] M. Alam, Y. Zhou, Strouhal numbers, forces and flow structures around two tandem cylinders of different diameters, Journal of Fluids and Structures 24 (4) (2008) 505-526.

[20] D. P. Lockard, M. R. Khorrami, M. M. Choudhari, F. V. Hutcheson, T. F. Brooks, D. J. Stead, Tandem Cylinder Noise Predictions, in: 13th AIAA/CEAS Aeroacoustics Conference, 2007.

[21] D. Sumner, Two circular cylinders in cross-flow: A review, Journal of Fluids and Structures 26 (6) (2010) 849-899.

[22] L. Jenkins, M. Khorrami, M. Choudhari, , C. McGinley, Characterization of unsteady flow structures around tandem cylinders for component interaction studies in airframe noise, in: AIAA-2005-2812, 2005.

[23] L. Jenkins, D. Neuhart, C. McGinley, M. Choudhari, Measurements of Unsteady Wake Interference Between Tandem Cylinders, in: Paper No. AIAA-2006-3202, 36th AIAA Fluid Dynamics Conference and Exhibit, 2006.

[24] N. D. H., J. L.N., C. M. M., K. M. R., Measurements of the flowfield interaction between tandem cylinders, in: AIAA Paper 20093275, 2009.

[25] M. R. Khorrami, M. M. Choudhari, D. P. Lockard, L. N. Jenkins, C. McGinley, Unsteady Flow Field Around Tandem Cylinders as Prototype Component Interaction in Airframe Noise, AIAA Journal 45, 8 (2007) 1930-1941.

[26] D. P. Lockard, M. M. Choudhari, M. R. Khorrami, D. H. Neuhart, F. V. Hutcheson, T. F. Brooks, D. J. Stead, Aeroacoustic Simulations of Tandem Cylinders with Subcritical Spacing, in: Paper No. AIAA-2008-2862, 14th AIAA/CEAS Aeroacoustics Conference, 2008. 
[27] C. Doolan, Flow and Noise Simulation of the NASA Tandem Cylinder Experiment using OpenFOAM, in: 15th AIAA/CEAS Aeroacoustics Conference, AIAA/CEAS, Miami, Florida, AIAA-2009-3157, 2009.

[28] D. Leclercq, C. Doolan, The interaction of a bluff body with a vortex wake, Journal of Fluids and Structures 25 (5) (2009) 867-888.

[29] M. Weinmann, R. D. Sandberg, C. D. Doolan, Flow and noise predictions for a tandem cylinder configuration using novel hybrid rans/les approaches, in: 16th CEAS/AIAA Aeroacoustics Conference, Stockholm, Sweden, 07 - 09 Jun 2010.

[30] A. Uzun, M. Y. Hussaini, An application of delayed detached eddy simulation to tandem cylinder flow field prediction, Computer \& Fluids 60 (2012) 71-85.

[31] G. A. . Bres, D. . Freed, M. . Wessels, S. . Noelting, F. . Perot, Flow and noise predictions for the tandem cylinder aeroacoustic benchmark, Physics of Fluids 24 .

[32] S. Fu, W. Haase, S. Peng, D. Schwamborn, Progress in Hybrid RANS-LES Modelling: Papers Contributed to the 4th Symposium on Hybrid RANS-LES Methods, Beijing, China, September 2011, Notes on Numerical Fluid Mechanics and Multidisciplinary Design, Springer, 2012.

URL http://books.google.co.uk/books?id=08TCNMZdG1MC

[33] Z. Xiao, J. Liu, J. Huang, S. Fu, Numerical dissipation effects on massive seperation around tandem cylinders, AIAA Journal Vol. 50, No 5 (2012) 1119 1136 .

[34] D. P. Lockard, Summary of the Tandem Cylinder Solutions from the Benchmark problems for Airframe Noise Computations-I Workshop, in: Proceedings of the 49th Aerospace Sciences Meeting Including the New Horizons Forum and Aerospace Exposition, AIAA-2011-0353, 2011.

[35] Proceedings of the workshop on Benchmark problems for Airframe Noise Computations (BANC), Stockholm, Sweden.

[36] D. Schwamborn, M. Strelets, Ataac - an eu-project dedicated to hybrid rans/les methods, in: in S. Fu et al. (Eds.): Progress in Hybrid RANS-LES Modelling, NNFM 117, pp. 59-75, 2012.

[37] D. Schwamborn, Results and lessons learned from the eu-project ataac, in: ERCOFTAC Symposium Unsteady Separation in Fluid-Structure Interaction, Mykonos, Greece, 17. - 21. June 2013.

[38] M. L. Shur, P. R. Spalart, M. K. Strelets, A. K. Travin, A hybrid RANS/LES approach with delayed-DES and wall-modelled LES capabilities, International Jounral of Heat and Fluid Flow 29 (2008) 1638-1649.

[39] F. R. Menter, Y. Egorov, The Scale-Adaptive Simulation Methodology for Unsteady Turbulent Flow Predictions. Part 1: Theory and Model Description, Flow Turbulence and Combustion 85 (2010) 113-138. 
[40] F. R. Menter, Two-Equation Eddy-Viscosity Turbulence Models for Engineering applications, AIAA Journal 32, 8 (1994) 1598-1604.

[41] F. R. Menter, Improved Two-Equation K- $\omega$ Turbulence Models for Aerodynamic Flows, NASA Technical Memorandum 103975.

[42] M. Weinmann, R. D. Sandberg, Suitability of Explicit Algebraic Stress Models for predicting complex three-dimensional flows, in: 19th AIAA Computational Fluid Dynamics, San Antonio, Texas. AIAA-2009-3663, 2009.

[43] S. Wallin, A. V. Johansen, An explicit algebraic Reynolds stress model for incompressible and compressible turbulent flows, Journal of Fluid Mechanics 403 (2000) 89-132.

[44] C. G. Speziale, S. Sarkar, T. B. Gatski, Modelling the pressure-strain correlation of turbulence: an invariant dynamical systems approach, Journal of Fluid Mechanics 227 (1991) 245-272.

[45] A. Hellsten, New Two-Equation Turbulence Model for Aerodynamic Applications, Ph.D. thesis, Helsinki University of Technology, Laboratory of Aerodynamics (2004).

[46] C. Hirsch, Numerical Computation of Internal and External Flows. Volume 1: Fundamentals of Numerical Discretisation, John Wiley \& Sons Ltd, 1994.

[47] A. Travin, M. Shur, M. Strelets, P. R. Spalart, Physical and Numerical Upgrades in the Detached-Eddy Simulation of Complex Turbulent Flows, in: R. Moreau, R. Friedrich, W. Rodi (Eds.), Advances in LES of Complex Flows, Vol. 65 of Fluid Mechanics and Its Applications, Springer Netherlands, 2004, pp. 239-254.

[48] Y. Kaneda, T. Ishihara, M. Yokokawa, K. Itakura, A. Uno, Energy dissipation rate and energy spectrum in high resolution direct numerical simulation of turbulence in a periodic box, Physics of Fluids 12,1 (2003) L21-L24.

[49] M. Weinmann, Simulations strategies for complex turbulent flows, Ph.D. thesis, University of Southampton (2011).

[50] N. Curle, The influence of solid boundaries on aerodynamic sound, Proc. Roy. Soc. London A231 (1955) 505-514.

[51] J. Seo, Y. Moon, Aerodynamic noise prediction for long-span bodies, Journal of Sound and Vibration 306 (2007) 564-579.

URL

http://linkinghub.elsevier.com/retrieve/pii/S0022460X07004130

[52] OpenFOAM, OpenCFD Ltd - OpenFOAM: http://www.opencfd.co.uk/.

[53] H. G. Weller, G. Tabor, H. Jasak, C. Fureby, A tensorial approach to computational continuum mechanics using object-oriented techniques, Computers in Physics 12, 6 (1998) 620-631.

[54] P. R. Spalart, C. L. Rumsey, Effective inflow conditions for turbulence models in aerodynamic calculations, AIAA Journal 45, 10 (2007) 2544-2553. 
[55] G. Schewe, On the force fluctuations acting on a circular cylinder in crossflow from subcritical up to transcritical Reynolds numbers, Journal of Fluid Mechanics 133 (1983) 265-285.

[56] Z. Xiao, J. Liu, J. Huang, S. Fu, Comparisons of three improved des methods on unsteady flows past tandem cylinders, in: in S. Fu et al. (Eds.): Progress in Hybrid RANS-LES Modelling, NNFM 117, pp. 59-75, 2013. 